\title{
Passive Near-Field Microscopy in Long-Wavelength Infrared*
}

\author{
Yusuke Kajihara, ${ }^{\dagger}$ Keishi Kosaka, and Susumu Komiyama \\ Department of Basic Science, The University of Tokyo, \\ Komaba 3-8-1, Meguro-ku, Tokyo 153-8902, Japan \\ (Received 10 November 2010; Accepted 3 March 2011; Published 9 April 2011)
}

\begin{abstract}
We have demonstrated ultrasensitive near-field microscopy in the long-wavelength infrared region without any external illumination. A scattering-type scanning near-field optical microscope was developed with a highly sensitive detector (charge sensitive infrared phototransistor: wavelength $\lambda \sim 14.5 \mu \mathrm{m}$ ) and a thermal evanescent wave was passively obtained from room-temperature objects by vertically modulating a tungsten probe. The spatial resolution of the near-field microscope was estimated to be better than $100 \mathrm{~nm}(\lambda / 100)$. The experimental results suggest that thermally excited surface plasmons on $\mathrm{Au}$ and surface phonons on $\mathrm{SiC}$ could be observed with our microscope. [DOI: 10.1380/ejssnt.2011.173]
\end{abstract}

Keywords: Light scattering; Photon emission; Surface waves; Plasmons; Phonons; Scattering-type scanning near-field optical microscopy (s-SNOM); Long-wavelength infrared (LWIR)

\section{INTRODUCTION}

Scattering-type scanning near-field optical microscopes (s-SNOMs) achieve sub-micrometer spatial resolution and represent a powerful measurement method in many spectral ranges [1-3]. Conventional s-SNOMs utilize a strong external light source to illuminate a metal probe tip and detect scattered photons induced by the sample-probe interaction. In this study, our objective was to carry out passive near-field microscopy without the use of such an external light source. Passive near-field microscopy can obtain a spontaneously activated evanescent field on material surfaces as shown in Fig. 1(a). The long-wavelength infrared (LWIR, wavelength $\lambda$ : 8-15 $\mu \mathrm{m}$ ) region contains many important spectra, such as molecular vibrations and lattice phonons. Hence, passive near-field microscopy in the LWIR region has the potential to visualize interesting phenomena in matter on the nanometer scale. However, this is a challenge since extremely weak radiation has to be detected. It has been reported that near-field like signals from hot samples $(443 \mathrm{~K})$ were obtained with an s-SNOM equipped with a mercury cadmium telluride (MCT) detector [4]. However, passive near-field imaging from room temperature objects has not yet been reported.

In this study, we developed an ultrahigh sensitivity sSNOM in the LWIR region by introducing an ultrahigh sensitivity LWIR detector, the charge sensitive infrared phototransistor (CSIP, $\lambda=14.5 \pm 0.5 \mu \mathrm{m}$ ) $[5,6]$. The CSIP shows more than $10^{2}$ times higher sensitivity than conventional LWIR sensors such as MCT detectors [7] and Quantum Well Infrared Photodetectors (QWIPs) [8]. Our S-SNOM consists of a probe control system and an LWIR microscope equipped with the CSIP. We studied $\mathrm{Au} / \mathrm{SiC}$ surfaces at room temperature and obtained passive nearfield signals with a spatial resolution better than $100 \mathrm{~nm}$. Strong signals from $\mathrm{Au}$ are ascribed to thermally activated surface plasmons. We also obtained near-field signals originating from surface phonons from $\mathrm{SiC}$.

\footnotetext{
*This paper was presented at the 6th International Workshop on Nano-scale Spectroscopy and Nanotechnology (NSS-6), Kobe University Centennial Hall, Kobe, Japan, 25-29 October, 2010.

†Corresponding author: kaji@thz.c.u-tokyo.ac.jp
}

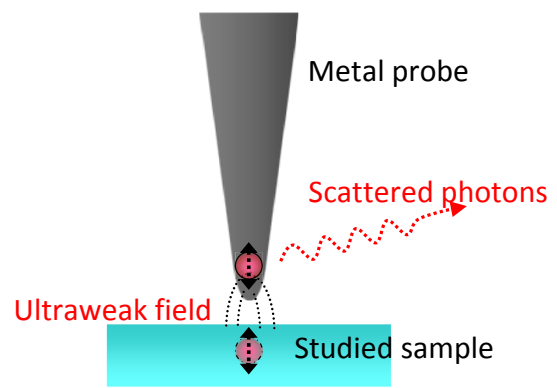

FIG. 1: Basic concept of passive near-field microscopy.

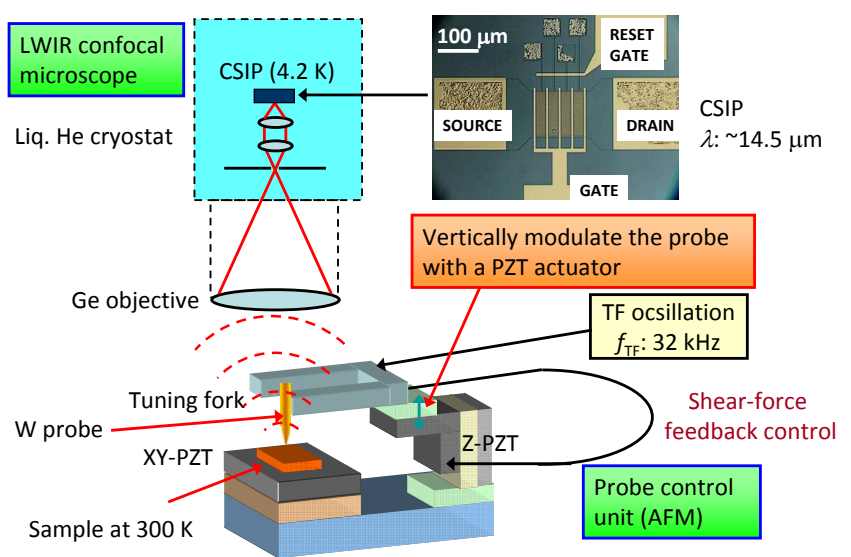

FIG. 2: Schematic diagram of the s-SNOM developed.

\section{PASSIVE NEAR-FIELD MICROSCOPE}

Figure 2 shows a schematic diagram of the s-SNOM developed by us. The s-SNOM consists of a probe control system and an LWIR confocal microscope equipped with a CSIP $(\lambda=14.5 \pm 0.5 \mu \mathrm{m})[9,10]$. The upper right in Fig. 2 shows an image of the CSIP detector with a $125 \mu \mathrm{m}$ $\times 125 \mu \mathrm{m}$ photosensitive area [5]. A tungsten probe is fabricated via electrochemical etching and has an apex radius smaller than $100 \mathrm{~nm}$. The probe is glued to one tine of a tuning fork. The probe-sample distance is nonoptically controlled in a shear-force mode [11] with $1 \mathrm{~nm}$ order accuracy. The mechanical resonance frequency of 


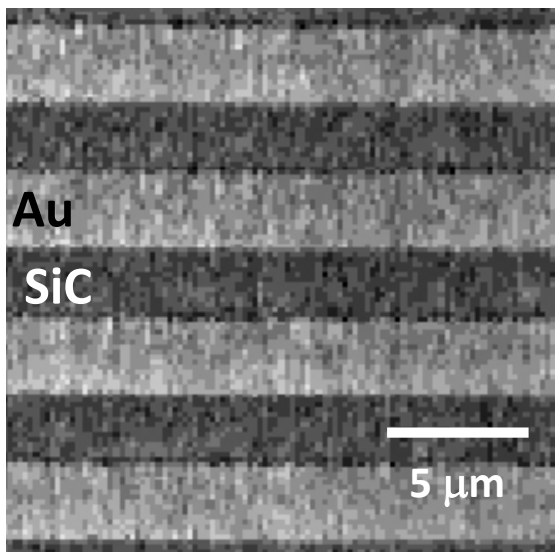

FIG. 3: Passive near-field image of $\mathrm{Au} / \mathrm{SiC}$ gratings.

the tuning fork is $f_{\mathrm{TF}} \sim 32 \mathrm{kHz}$ with a quality factor of $\sim 1500$.

Thermal emission from a sample at room temperature $(300 \mathrm{~K})$ is scattered by the probe apex and is detected by the CSIP via a confocal system [9]. The CSIP is cooled to $4.2 \mathrm{~K}$ in a liquid helium cryostat to avoid thermal noise from the CSIP. The numerical aperture of a germanium objective lens is 0.60 and the diameter of a confocal pinhole is $62.5 \mu \mathrm{m}$. A ZnSe window is placed between the $4.2 \mathrm{~K}$ region and the $300 \mathrm{~K}$ region. During operation, the obtained signals contain a large amount of far-field background and small near-field components. To extract only the near-field components, we vertically modulate the probe with a PZT actuator and demodulate the signals with a lock-in amplifier. In conventional s-SNOMs, the probe is operated in a tapping mode, where the probe tip is vertically modulated with respect to the sample surface at $f_{\mathrm{TF}}$, and the near-field component is detected by demodulating the optical signal $[3,12]$. In this study, however, the modulation frequency was lower than $1 \mathrm{kHz}$ for higher performance of the CSIP. The height of the probe was hence modulated at a frequency of $f_{\mathrm{M}}=10 \mathrm{~Hz}$, independent of the resonant lateral oscillation of the tuning fork at $f_{\mathrm{TF}} \sim 32 \mathrm{kHz}$. When the probe scanned the sample, the bottom height of the probe was kept around $10 \mathrm{~nm}$ by sample-holding the tuning fork current at the bottom. Passive near-field signals were thus obtained by scanning the modulated probe over the sample surface. At the same time, passive far-field signals were obtained without signal demodulation.

\section{PASSIVE NEAR-FIELD SIGNALS}

Figure 3 shows a passive near-field image on $3 \mu \mathrm{m}$-pitch $\mathrm{Au} / \mathrm{SiC}$ gratings, in which $\mathrm{Au}$ was deposited $100 \mathrm{~nm}$ on the $\mathrm{SiC}$ substrate. This image was obtained from a $20 \mu \mathrm{m}$ $\times 20 \mu \mathrm{m}$ area by scanning the modulated probe without any external illumination. The scan step was $200 \mathrm{~nm}$ and the scan interval was $300 \mathrm{~ms}$ for each step (this took a total time of around 1 hours). In this image, Au and $\mathrm{SiC}$ are clearly distinguished, even though the spatial resolution of far-field microscopy was limited to $15 \mu \mathrm{m}$ [9] because of the diffraction limit. Figure 4 shows a one dimensional

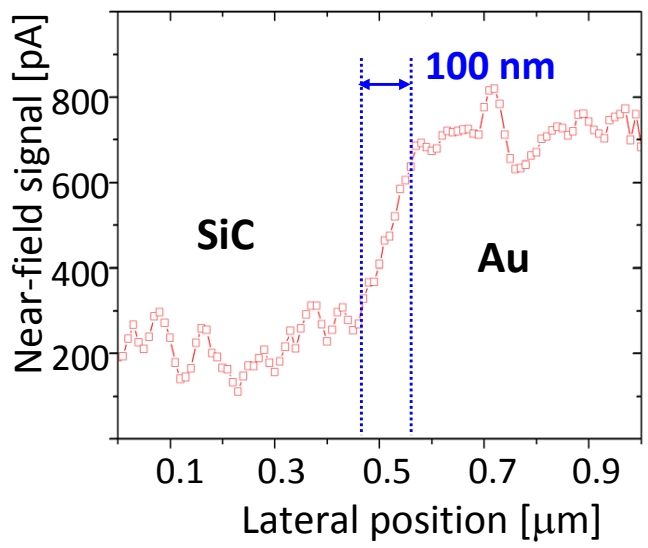

FIG. 4: Estimation of spatial resolution.
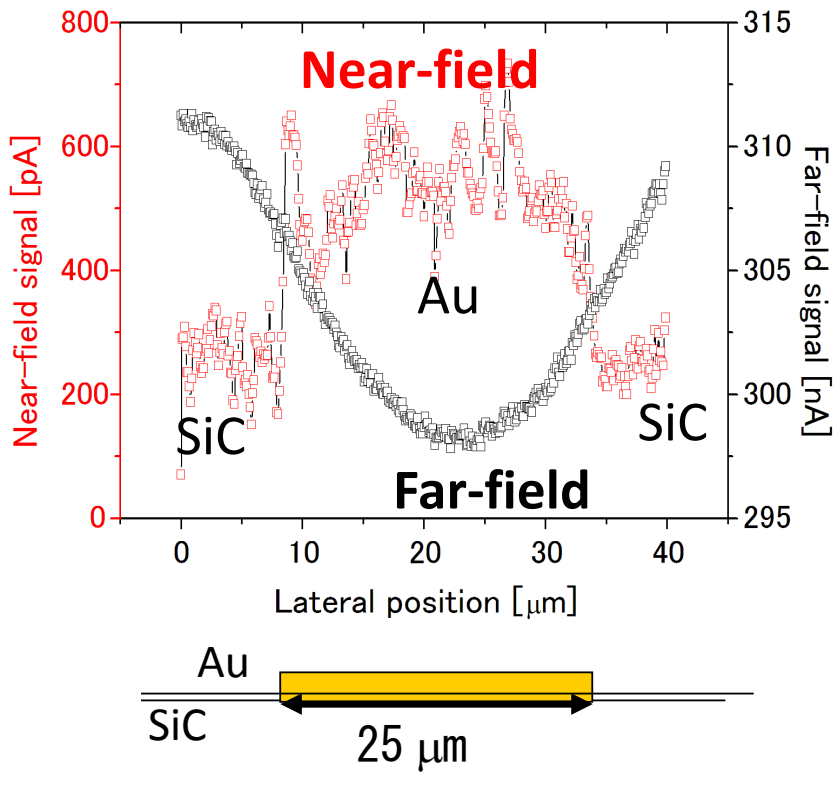

FIG. 5: One dimensional profiles of passive far-field signals and near-field signals.

profile of the $\mathrm{Au} / \mathrm{SiC}$ edge with a scan step of $10 \mathrm{~nm}$. From these results, the spatial resolution of our passive $\mathrm{s}-\mathrm{SNOM}$ is estimated to be better than $100 \mathrm{~nm}(\lambda / 150)$.

Figure 5 displays one dimensional profiles of both farfield and near-field signals. These were obtained from a sample consisting of a SiC substrate and $25 \mu$ m-width $\mathrm{Au}$ (100 nm thickness). In Fig. 5, the near-field signal from $\mathrm{Au}$ is larger than that from SiC, whereas the far-field signal from $\mathrm{Au}$ is smaller than that from $\mathrm{SiC}$. The contrasts of the near-field and far-field signals are completely opposite. In addition, the near-field signal intensity is around 0.002 of the far-field signal intensity although the detection area of the near-field microscopy was around 0.00005 of that of the far-field microscopy. These findings indicate that the signal origins of the far-field and near-field are different.

Here, we interpret the experimental results. First, the passive far-field signal is computed by multiplying the emissivity of the material by the black body radiation (BBR) [9]. In this measurement, the calculated BBR val- 
ues of $\mathrm{Au}$ and $\mathrm{SiC}$ are the same because of their identical temperatures. The emissivity of $\mathrm{SiC}$ is around 0.60 [13], whereas the emissivity of $\mathrm{Au}$ is almost zero. Thus, the far-field signal from SiC should be higher and the characteristics of the far-field signals in Fig. 5 are reasonable.

On the other hand, the near-field result suggests that a strongly localized field exists at the Au surface. As shown in previous work, such as Ref. [14], over the metal surface, the local density of states at a height of less than $100 \mathrm{~nm}$ is much higher than the density of states at a height of $1 \mu \mathrm{m}$ in the LWIR region. This theoretical analysis indicates that a strongly localized electromagnetic field exists at the metal surface in the LWIR region. The localized components at the metal surface are thermally excited surface plasmons (SPs) [15]. It is impossible to observe such localized components from far-field measurements. However, by scattering the localized waves with a metal probe, these components can be revealed. To the best of our knowledge, only our passive near-field microscopy technique can observe such thermally excited SPs at room temperature.

Finite signals are also seen at the $\mathrm{SiC}$ surface in Fig. 5. The origin of these signals is likely surface phonons (SPhs) because lattice vibrations can excite large polarizations in ionic crystals such as $\mathrm{SiC}$. The resonance frequency of $\mathrm{SPh}$ is inside the Reststrahlen band between the transverse optical (TO) and the longitudinal optical (LO) phonon frequencies, $\omega_{\mathrm{T}}$ and $\omega_{\mathrm{L}}$ [14]. Here, the Reststrahlen band of $\mathrm{SiC}$ is from $\omega_{\mathrm{L}}=968 \mathrm{~cm}^{-1}(10.3 \mu \mathrm{m})$ to $\omega_{\mathrm{T}}=797 \mathrm{~cm}^{-1}(12.5 \mu \mathrm{m})$. It is slightly far from the detectable wavelength of the CSIP $(14.5 \mu \mathrm{m} \pm 0.5 \mu \mathrm{m})$, which should yield smaller signals in Fig. 5. The surface phonon resonance is close to $\omega_{\mathrm{L}}$, as experimentally shown with active s-SNOMs [16] or the attenuated total reflection method [17]. We therefore investigated a GaN substrate with our passive near-field microscope. The LO phonon frequency $\omega_{\mathrm{L}}$ of $\mathrm{GaN}$ is $740 \mathrm{~cm}^{-1}(13.5 \mu \mathrm{m})$ and the TO phonon frequency $\omega_{\mathrm{T}}$ is $560 \mathrm{~cm}^{-1}(17.8 \mu \mathrm{m})$. Much larger signals should be obtained on GaN since the detectable frequency of the CSIP is very close to $\omega_{\mathrm{L}}$. However, we obtained no signal at all from the GaN substrate, which is completely opposite to our expectation. Further experimental and theoretical analyses are required to understand and explain this.

\section{CONCLUSIONS}

In this paper, we reported passive near-field microscopy in the LWIR region. We developed an s-SNOM consisting of a probe control system and a confocal microscope equipped with a CSIP detector $(\lambda: 14.5 \mu \mathrm{m} \pm 0.5 \mu \mathrm{m})$. Near-field signals were passively obtained from a roomtemperature object $(\mathrm{SiC} / \mathrm{Au})$ by vertically modulating a tungsten probe to suppress the far-field background. The spatial resolution of our s-SNOM was estimated to be better than $100 \mathrm{~nm}(\lambda / 150)$ by scanning a probe across the $\mathrm{SiC} / \mathrm{Au}$ edges. Large near-field signals from the $\mathrm{Au}$ surface are suggested to be due to thermally excited surface plasmons. In addition, finite near-field signals are obtained from the SiC surface, which should be ascribed to surface phonons. These experimental results confirm that our s-SNOM is a promising tool for studying mesoscopic phenomena, such as biomolecular protein interactions, in the future.

\section{Acknowledgments}

This work was supported by the CREST project of the Japan Science and Technology Agency (JST) and a Grant-in-Aid for Challenging Exploratory Research.
[1] F. Zenhausern, M. P. O'Boyle, and H. K. Wickramasinghe, Appl. Phys. Lett. 65, 1623 (1994).

[2] Y. Inouye and S. Kawata, Opt. Lett. 19, 159 (1994).

[3] B. Knoll and F. Keilmann, Nature 399, 134 (1999).

[4] Y. De Wilde, F. Formanek, R. Carminati, B. Gralak, P. Lemoine, K. Joulain, J. Mulet, Y. Chen, and J.-J. Greffet, Nature 444, 740 (2006).

[5] T. Ueda, Z. An, K. Hirakawa, and S. Komiyama, J. Appl. Phys. 103, 093109 (2008).

[6] P. Nickels, S. Matsuda, T. Ueda, Z. An, and S. Komiyama, IEEE J. Quantum Electronics 46, 384 (2010).

[7] P. Norton, Opto-electron. Rev. 10, 159 (2002).

[8] A. Rogalski, J. Appl. Phys. 93, 4355 (2003).

[9] Y. Kajihara, S. Komiyama, P. Nickels, and T. Ueda, Rev. Sci. Instrum. 80, 063702 (2009).

[10] Y. Kajihara, K. Kosaka, and S. Komiyama, Rev. Sci. Instrum. 81, 033706 (2010).
[11] K. Karrai, and R. D. Grober, Appl. Phys. Lett. 66, 1842 (1995).

[12] B. Knoll and F. Keilmann, Opt. Commun. 182, 321 (2000).

[13] C. P. Cagran, L. M. Hanssen, M. Noorma, A. V. Gura, and S. N. Mekhontsev, Int. J. Thermophys. 28, 581 (2007)

[14] K. Joulain, J.-P. Mulet, F. Marquier, R. Carminati, and J.-J. Greffet, Surf. Sci. Rep. 57, 59 (2005).

[15] H. Raether, Surface Plasmons on Smooth and Rough Surfaces and on Gratings (Springer-Verlag, Berlin, 1988).

[16] R. Hillenbrand, T. Taubner, and F. Keilmann, Nature 418, 159 (2002).

[17] S. S. Ng, Z. Hassan, and H. Abu Hassan, Appl. Phy. Lett. 91, 081909 (2007). 